\title{
The Effect of Softener in Polyester Textile Fibers to the Pruritus Response and Erythema with Atopic Dermatitis Patients
}

\author{
Rahmat Hidayat $^{1}$, Alwi Andi Mappiasse ${ }^{1}$, Farida Tabri ${ }^{1}$, Yohanes Widodo ${ }^{2}$, Firdaus Hamid ${ }^{3}$, \\ Raden Satriono ${ }^{4}$
}

${ }^{1}$ Department of Dermatology and Venereology, Medical Faculty, Hasanudin University, Makassar, Indonesia

${ }^{2}$ Department of Dermatology and Venereology, Medical Faculty, Gajah Mada University, Yogyakarta, Indonesia

${ }^{3}$ Department of Microbiology, Medical Faculty, Hasanudin University, Makassar, Indonesia

${ }^{4}$ Department of Pediatric, Medical Faculty, Hasanuddin University, Makassar, Indonesia

Email address:

data_dayat@outlook.com (R. Hidayat)

\section{To cite this article:}

Rahmat Hidayat, Alwi Andi Mappiasse, Farida Tabri, Yohanes Widodo, Firdaus Hamid, Raden Satriono. The Effect of Softener in Polyester Textile Fibers to the Pruritus Response and Erythema with Atopic Dermatitis Patients. American Journal of Clinical and Experimental Medicine. Vol. 5, No. 6, 2017, pp. 186-189. doi: 10.11648/j.ajcem.20170506.11

Received: February 21, 2017; Accepted: March 4, 2017; Published: September 25, 2017

\begin{abstract}
Atopic dermatitis is a chronic skin disease that most commonly occurs in children. This study aimed to assess the effective of softener on polyester textile material on the pruritus degree and erythema of patients with atopic dermatitis. The research was conducted at Senior High School 17 of Makassar. The method used was clinical test with experimental design to determine the role of softener on pruritus response of patients with atopic dermatitis. The sample consisted of 50 people given fabric softener material on the right and without softener on the left arms. Two hours and twenty four hours later the degree of pruritus and erythema was measured. The results of the research indicate that the use of softener on polyester textile increases the degree of pruritus and erythema.
\end{abstract}

Keywords: Atopic Dermatitis, Erythema, Pruritus, Softener

\section{Introduction}

Atopic dermatitis (AD) is a chronic recurrent skin disease that generally occurs most often during infancy and children, though in fact be informed of all ages and can last a lifetime. Atopic dermatitis is usually found from the age of 2 months and about 1 year in $60 \%$ of patients, $30 \%$ seen for the first time at the age of 5 years, and only $10 \%$ of atopic dermatitis arises between the ages of 6 to 20 years [1]. As many as $60 \%$ of parents who have $\mathrm{AD}$, have children who are also suffering from the same disease. The prevalence in children is high, which is around $80 \%$ if both parents have from $\mathrm{AD}$ [2]. According to the International Study of asthma and Allergies in Children, the prevalence of AD in children varies in different countries. The prevalence of $\mathrm{AD}$ in children in Iran and China about $2 \%$ and $20 \%$ in Australia, England and Scandinavia. The high prevalence was also obtained in the
United States in the amount of $17.2 \%$ [3].

Several studies have measured morbidity AD obtained cases often reach the highest morbidity score compared to other diseases. Besides reduction in quality of life is directly related to the severity of the AD. Psychological morbidity associated with continuous scratching, disturbed sleep, and scars visible on the skin can also affect patients [4].

Atopic dermatitis is not a skin disorder caused by one type of cause. This situation is the result of a complex interaction of many factors such as: 1) the susceptibility of genetic, 2) exposure to allergens, irritants, or a change in the weather coming from the environment, 3) dysfunction barrier of the skin, 4) stressors, psychological, and 5) abnormal patterns of immunological reactions, This complex interaction can cause an allergic reaction becomes a factor that plays an important role in a patient, but on the other patient factors that may play a bigger role is a disorder of the skin barrier function, infection or physical stressors / psychological [3]. 
Pruritic AD triggered by a variety of endogenous and exogenous factors through the free end of the non-specific myelin $\mathrm{C}$ fibers. Some reports indicate that the skin's immune system is closely related to provocation and intensity of pruritus in patients with AD. Atopic dermatitis patient's skin reduces pruritus threshold and a long duration of pruritus in pruritic stimuli compared with healthy skin, where it is possible to produce a higher tendency to occur pruritus during stimulation [5].

The skin is an organ that most outside that protect the body from the surrounding environment and also helps the body to interact with the environment. In patients with $\mathrm{AD}$ a state of hypersensitivity to external factors such as the materials used clothes made of wool, nylon, polyester, which if exposed again will give a reaction, first response occurs early phase (early phase) followed by the slow phase (late phase reaction), Macroscopic picture seen in the slow phase is diffuse edema, erythema, induration, and itch a little bit. Efforts to reduce pruritus $\mathrm{AD}$ patients have been conducted using zinc oxide is added to the textile material, rapid improvements in the severity of the $\mathrm{AD}$, pruritus, and sleep quality of patients $\mathrm{AD}$. This is possible because the high antioxidant than zinc oxide, as well as antibacterial activity [6].

A previous study by Katayama et al (2011), conducted in developing countries found that $10-20 \%$ of children suffer from AD. Atopic dermatitis prevalence in children of Asian ethnicity has not been widely reported. The prevalence rate ever reported was $20.1 \%$ in Hong Kong, $19 \%$ in Japan and $20.8 \%$ in Singapore [6].

Assessment of the degree of pruritus can be done by using a scale Visual Analogue Scale (VAS) which uses the numbers 0 to 10 to describe the level of pruritus [7].

The effect of Softener on polyester textile material on the response of patients with pruritus $\mathrm{AD}$ unknown. Under these conditions, the study aims to assess the effectiveness of softener on polyester textile material on the degree of pruritus and erythema patients with atopic dermatitis.

\section{Materials and Methods}

\subsection{Location and Research Time}

The study was conducted at Senior High School 17 of Makassar.

\subsection{Design Study}

This study used two groups-blind clinical trials. The research variables consist of: the independent variable (softener), the dependent variable (the degree of pruritus, erythema), and the control variables (patients with atopic dermatitis).

\subsection{Procedure}

The study population is the student / Senior High School 17 of Makassar with a history of atopic dermatitis. The samples were all patients who met the inclusion criteria $\mathrm{AD}$, willing to participate in the study by signing an informed consent.

Selection of patients who meet the inclusion and exclusion criteria. Data retrieval include: History: The identity of the patient, the course of disease, a history of atopic (fill in the questionnaire). Patients described first study procedures, ways of working and the side effects that may occur during treatment. Patients signed informed consent. Physical examination performed on all subjects of research. Pruritus induced occlusive polyester textile material test was performed in all study subjects. Assessment of erythema and pruritus degree by VAS after 2 hours and 24 hours and documentation by Image capture.

\subsection{Analysis of Data}

Processing data using the Statistical program for Social Science (SPSS) version 22. The data have been obtained will be statistically tested with the Mann-Whitney test and the Wilcoxon Signed Rank Test. The results of hypothesis testing is said to be significant if $p<0.05$. The data was processed and presented in tabular form and narrative.

\section{Results}

Studies have been conducted using two groups-blind clinical trial to assess the effect of the softener on polyester textile material on the degree of pruritus patients with atopic dermatitis. This research was conducted in Senior High School 17 of Makassar. When the study for two weeks is from 1 to 12 August 2016, with the research subjects were patients with atopic dermatitis, met the inclusion criteria, willing to participate in the study by signing an informed consent. During the period of study, study subjects collected as many as 50 people with atopic dermatitis, in which three men and 47 women. Patients in the age group 16 years as many as 18 people (36\%), age group 17 years as many as 23 people (46\%) and the age group 18 years as many as 9 people $(9 \%)$.

Analysis of the influence on the degree of pruritus cause softener with VAS assessment showed that for the measurement time 2 hours there is a significant difference in mean VAS according to group $(\mathrm{p}<0.001)$. Mean VAS administration group softener on polyester textile materials significantly higher than the group that was not given a softener, which is 3.46 to 2.00 . For the measurement time 24 hours, there is a significant difference in mean VAS according to group $(\mathrm{p}<0.001)$. Mean VAS softener group was significantly higher than among non softener, which is 5.44 to 3.82 (Table 1 ).

Table 1. Comparison of Mean VAS by Group.

\begin{tabular}{llllll}
\hline VAS & Group & $\mathbf{n}$ & Mean & SD & $\mathbf{p}^{\mathbf{1}}$ \\
\hline \multirow{2}{*}{2 Hours } & Softener & 50 & 3,46 & 1,16 & \multirow{2}{*}{20,001} \\
& Non softener & 50 & 2,00 & 1,29 & \\
& Softener & 50 & 5,44 & 1,80 & $<0,001$ \\
\hline
\end{tabular}

Mann-Whitney test 
Comparison of the mean erythema according to the group obtained, for 2 hours of measurement time there are significant differences in the mean erythema according to group $(\mathrm{p}<0.001)$. Mean erythema for group administration softener on polyester textile materials significantly higher than the group that was not given a softener, which is 1.12 to 0.68 . For the measurement time 24 hours, there is a significant difference in mean erythema according to group $(p<0.001)$. Mean VAS softener group was significantly higher than among non softener, which is 1.74 to 1.32 (Table 2).

Table 2. Comparison of Mean Erythema by Group.

\begin{tabular}{llllll}
\hline Erythema & Group & $\mathbf{n}$ & Mean & SD & $\mathbf{p}^{\mathbf{1})}$ \\
\hline \multirow{2}{*}{2 Hours } & Softener & 50 & 1,12 & 0,44 & $<0,001$ \\
& Non softener & 50 & 0,68 & 0,47 & \\
\multirow{2}{*}{24 Hours } & Softener & 50 & 1,74 & 0,44 & $<0,001$ \\
& Non softener & 50 & 1,32 & 0,59 & \\
\hline
\end{tabular}

Mann-Whitney test

Comparison of mean VAS by time measurements show that the group given softener on polyester textile materials, there is a difference between the mean VAS measurement time of 2 hours to 24 hours $(p<0.001$ ). Mean VAS at 24 hours was significantly higher than at 2 hours, ie 5.44 to 3.46. In the group that was not given softener, there is a difference between the mean VAS measurement time of 2 hours to 24 hours $(p<0.001)$. Mean VAS at 24 hours was significantly higher than at 2 hours, which is 3,82 to 2.00 (Table 3).

Table 3. Comparison of Mean VAS by Time Measurement

\begin{tabular}{llllll}
\hline Group & Time & $\mathbf{n}$ & Mean & SD & $\mathbf{p}^{\mathbf{1 )}}$ \\
\hline \multirow{2}{*}{ Softener } & 2 Hours & 50 & 3,46 & 1,16 & $<0,001$ \\
& 24 Hours & 50 & 5,44 & 1,80 & \\
\multirow{2}{*}{ No softener } & 2 Hours & 50 & 2,00 & 1,29 & $<0,001$ \\
& 24 Hours & 50 & 3,82 & 1,70 & \\
\hline
\end{tabular}

Wilcoxon Signed Rank test

Comparison of the mean erythema by time measurements show that the group given softener on polyester textile materials, erythema mean there is a difference between the measurement time 2 hours to 24 hours $(\mathrm{p}<0.001)$. The mean erythema at 24 hours was significantly higher than at 2 hours, ie 1.74 to 1.12 . In the group that was not given softener, erythema mean there is a difference between the measurement time 2 hours to 24 hours ( $p<0.001$ ). Mean VAS at 24 hours was significantly higher than at 2 hours, ie 1.32 to 0.68 (Table 4).

Table 4. Comparison of Mean Erythema by Time Measurement.

\begin{tabular}{llllll}
\hline Group & Time & n & Mean & SD & $\mathbf{p}^{\mathbf{1}}$ \\
\hline \multirow{2}{*}{ Softener } & 2 Hours & 50 & 1,12 & 0,44 & \multirow{2}{*}{ No softener } \\
& 24 Hours & 50 & 1,74 & 0,44 & $<0,001$ \\
& 2 Hours & 50 & 0,68 & 0,47 & $<0,001$ \\
& 24 Hours & 50 & 1,32 & 0,59 & \\
\hline
\end{tabular}

Wilcoxon Signed Rank test
Comparison of mean vase delta and delta mean erythema according to the group if the difference delta shows the mean VAS compared between the two groups, it was not found significant differences in mean delta between the group given a softener on polyester textile materials $(5.44$ to $3.46=1.98$ ) and a group that was not given softener $(3.82$ to $2.00=1.82)$ with $\mathrm{p}=0.300$. Similarly, delta mean erythema also did not reveal significant differences between the groups were given a softener $(1.74$ to $1.12=0.62)$ and the group that was not given softener $(1.32-.68=0.64)$ with $\mathrm{p}=0.887$ (appendix, Table 5).

Table 5. Comparison of Mean Delta VAS and Mean Delta Erythema by Group.

\begin{tabular}{llllll}
\hline Variable & Group & $\mathbf{n}$ & Mean & SD & $\mathbf{p}^{\mathbf{1})}$ \\
\hline \multirow{2}{*}{ VAS } & Softener & 50 & 1,98 & 1,39 & \multirow{2}{*}{0,300} \\
& Non softener & 50 & 1,80 & 1,73 & \\
\multirow{2}{*}{ Erythema } & Softener & 50 & 0,62 & 0,57 & \multirow{2}{*}{0,887} \\
& Non softener & 50 & 0,64 & 0,72 & \\
\hline
\end{tabular}

Mann-Whitney test

\section{Discussion}

This study shows that the use softener on polyester textile fibers increase the degree pruritus and erythema. Atopic dermatitis typically affects infants and children, but not infrequently also affects adults in the United States, Europe, Japan, Australia and other industrialized countries, the prevalence of atopic dermatitis in children reach 10-20\%, whereas in adults is approximately $1-3 \%$. Where the prevalence of atopic dermatitis in developing countries is lower than the developed countries. Dermatitis most often appears in the first year of life, usually after the age of two months. While in general, atopic dermatitis prolonged adolescence or adulthood, then tended to decline and improved (recovered) after the age of 30 years, only a small fraction continues into old age [1].

Atopic dermatitis is a chronic inflammatory disease of the skin that usually begins during the first year of life. In the management of the DA, the correct approach requires a combination of several management to identify and eliminate the trigger factors, and enhance the skin barrier changes [8-10].

Atopic dermatitis can be triggered or exacerbated the situation by various factors such as; dryness of the skin due to various things (psychological stress, changes in temperature or humidity, skin infections by Staphylococcus aureus, contact with irritant materials (wool, nylon, polyester), exposed to materials aeroallergens or food allergens [11].

The incidence of AD in women more than men with a ratio of 1.3: 1 was found ranging from the ages of two months and about one year in $60 \%$ of patients, $30 \%$ at five years of age, and only $10 \%$ of atopic dermatitis arises between the ages of six to 20 years. Epidemiological studies of various countries show that in addition to genetic susceptibility factors observed in cases of familial and twin, AD prevalence continues to rise and reach the estimated $20 \%$ in the general 
population, the prevalence is influenced by racial differences and environmental factors. Studies in North America and Singapore found significant racial differences for events AD children [2]. Chinese immigrants in Hawaii and New Zealand experienced an increase in cases of $\mathrm{AD}$ significantly compared with the incidence of $\mathrm{AD}$ in his home country. This shows the role of environmental factors that influence the prevalence of $\mathrm{AD}$ [12].

The degree of pruritus on the measurement of 2 hours and 24 hours with VAS was higher in the group given softener compared with those not given softener. In this study, the mean VAS at 24 hours was higher than 2 hours both in the group given or not given clothes softener. In patients with $\mathrm{AD}$, immunological disorders whose main profile is the formation of excessive IgE, thus facilitating the occurrence of type I hypersensitivity and impaired regulation of cytokines. When the patient is exposed to a trigger factor (antigen). Precipitating factors such as fabric softener containing a mixture of hydrochloric alkyl dimethyl benzyl ammonium and a sensitizer material irritation especially in atopic dermatitis patients. These antigens will be bound $\operatorname{IgE}$ on the surface of mast cells and will cause the release of chemical mediators include histamine that cause itching. This reaction occurs usual 15-60 minutes after a person associated with precipitating factors. Longer exposure is directly proportional to the length of pruritus [13]. Percentage of erythema 2 hours and 24 hours of group given a softener is higher than the group that was not given softener. In patients with $\mathrm{AD}, \mathrm{Th} 2$ immune response occurs to stimuli exogenous factors, because of the genetic changes that lead to excessive production of IL-4. Th2 cells secrete IL-4, IL-5, IL-13 which led to an increase Ievel $\operatorname{IgE}$ and induces adhesion molecules which contribute to the inflmasi cell migration into the skin. Th2 cells stimulate B cells to produce IgE. Mast cells have been attached $\operatorname{IgE}$ will undergo degranulation to release mediators that cause the symptoms of inflammation in patients with $\mathrm{AD}$.

In the assessment of 2 hours and 24 hours in both groups are erythema erythema which spreads more positive on the assessment of 24 hours. In Atopic dermatitis occurs late phase reaction, namely local inflammatory reaction occurred after 2 hours of contact with the trigger factors such as fabric softener, which reached its peak hours of 6 to 12 and will remain there until 24-48 hours. The mechanism of the late phase inflammatory reaction caused by the influence or induction of cytokines that cause the expression of adhesion molecules on endothelial cells that infiltrate into the inflammation. As late phase reaction triggers mast cells that secrete TNF-alpha, IL-5, whereas Th2 cells that produce IL-3, IL-4, IL-5 and IL-13 will maintain the inflammation until 24-48 hours.

\section{Conclusions}

The researchers concluded that the use of clothing made from polyester can trigger and eritama pruritus in patients with atopic dermatitis. Pruritus and erythema degrees higher in the use of polyester fabric that use softener compared to the use of without softener in patients with atopic dermatitis. Researchers suggest that further research using other textile materials which cause irritation to the patient $\mathrm{AD}$, such as wool and nylon. Further research is required to use the active ingredient of the product, namely fabric softener; Quaternary Ammonium Compounds. As the management of patients with atopic dermatitis consideration in choosing clothes made of polyester and the use of fabric softener.

\section{References}

[1] Fieldman et al. (2014). Guidelines of Care for the Management of Atopic Dermatitis. Section 1. Diagnosis and Assessment of Atopic Dermatitis. J Am Acad Dermatol. 70: 338-51.

[2] Flohr \& Mann. (2014). New Insights into the Epidemiology of Childhood Atopic Dermatitis. Allergy. 69: 3-16.

[3] Friedmann et al. (2010). Atopic Dermatitis. In Bums T., Breathnach, S., Cox, N. \& Griffiths, C. (Eds.) Rook's Texbook Of Dermatology. $8^{\text {th }}$ ed. Victoria: Willey-Blackwell.

[4] Katayama et al. (2011). Japanese Guidelines for Atopic Dermatitis. Allergol Int. 60: 205-20.

[5] Peytavi \& Metz. (2012). Atopic Dermatitis in Children: Management of Pruritus. JEADV. 26: 2-8.

[6] Wiegand et al. (2009). Skin-Protective Affects of a Zinc Oxide-Functionalized Textile and its Relevance for Atopic Dermatitis. Clinical, Cosmetic and Investigational Dermatology.

[7] Reich et al. (2012). Visual Analogue Scale: Evaluation of the Instrument for the Assessment of Pruritus. Acta Derm Venereol. (92(5): 497-501.

[8] Haug et al. (2010). Coated Textiles in the Treatment of Atopic Dermatitis. Curr Probl Dermatol. 33: 144-51.

[9] Lazarrov. (2010). Textile Dermatitis in Patients With Contact Sensitization in Israel: a 4-Year Prospective Study. JEADV. 18: $531-37$.

[10] Lopes et al. (2013). Functional Textiles for Atopic Dermatitis: A Systematic Review and Meta-Analysis. Pediatr Allergy Immunol. 24: 603-13.

[11] Thomsen. (2014). Atopic Dermatitis: Natural History, Diagnosis, and Treatment. ISRN Allergy. 10: 1-7.

[12] Lee. (2014). Epidermal Permeability Barrier Defects and Barrier Repair Therapy in Atopic Dermatitis. Allergy Asthma Immunol Res. 4: 276-87.

[13] Biddenkotte \& Steinhoff. (2010). Pathophysiology and Therapy of Pruritus in Allergic and Atopic Diseases. Allergy. 65: 805-21. 